\title{
Intravenous Iron to Treat Anemia becomes an Essential Service to Conserve Blood during the COVID-19 Pandemic
}

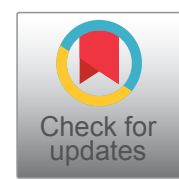

Obianuju Okocha, MD*, Betty M Luan-Erfe, MD, Jack M Peace, MD and Bobbie Jean Sweitzer, MD, FACP

Department of Anesthesiology, Northwestern University, Feinberg School of Medicine, Illinois, USA

\begin{abstract}
With the outbreak of Coronavirus Disease 2019 (COVID-19), blood drives have been cancelled, resulting in significantly fewer donations. However, blood components are still being used to treat patients with acute and chronic hemorrhage, trauma, cancer, hematologic disorders and even COVID-19 with disseminated intravascular coagulation. Consequently, national and local blood supplies will dwindle and may become critically limited. The clinician's stewardship in anemia management and blood use has become ever more important during this pandemic. Clinicians are responsible for the timely diagnosis of anemia, its treatable causes and prompt therapy with alternatives to blood transfusions. However, there are challenges during this pandemic with restrictions on travel with many institutions and practitioners limiting patient visits to prevent community spread of COVID-19.

To conserve the supply of blood components during the COVID-19 pandemic, proactive and efficient measures to diagnose anemia and timely treat with intravenous iron when indicated, while minimizing clinic visits are imperative. We advocate and share our experience with an anemia management process. Randomized controlled trials, cohort studies, and our institution's case examples demonstrate robust hemoglobin increases within as little as two weeks of treatment. Unfortunately, unsubstantiated concerns about the safety, costs and logistical challenges of administering intravenous iron have limited its use despite a safety profile similar to that of oral iron and superior to allogeneic blood transfusions. We argue that intravenous iron is integral to institutions' and clinicians' stewardship of blood supplies during the current shortage triggered by the COVID-19 pandemic.
\end{abstract}

\section{Glossary of Terms}

ASA-PS: American Society of Anesthesiologists Physical Status; CBC: Complete blood count; COVID-19: Coronavirus Disease 2019; eGFR: Estimated glomerular filtration rate; F: Female; FDA: Food and Drug Administration; Hb: Hemoglobin; IDA: Iron-deficiency anemia; IRB: Institutional Review Board; PPAE: Pre-procedure anemia evaluation; TIBC: Total iron binding capacity; TSAT: Transferrin saturation; TSH: Thyroid stimulating hormone; SARS-CoV-2: Severe acute respiratory syndrome coronavirus 2

\section{Introduction}

As a precautionary measure during the Coronavirus Disease 2019 (COVID-19) pandemic, thousands of community-based blood drives have been canceled due to restrictions on gatherings and stay-at-home directives [1,2]. These restrictions have led to reductions in blood donations and availability of blood components [2]. Packed red blood cells have a shelf-life of 35-42 days, depending on the preservative used [3], and the recommended time interval between whole blood donations is 8 weeks [4]. The American Red Cross estimates that someone in the United States receives blood every two seconds [4]. Perhaps this demand can be mitigated by wider adoption of recommended transfusion triggers. The American Red Cross issued an open letter projecting severe constraints on their inventory of red blood cells [1] and has encouraged blood donation at local blood banks amidst blood drive cancellations. Blood donors are critical to our medical infrastructure and there is growing concern that as the COVID-19 pandemic persists, potential donors, blood bank staff and resources will be affected [2]. Furthermore, although there have been no reported incidences of blood

*Corresponding author: Obianuju Okocha, MD, Department of Anesthesiology, Northwestern University, Feinberg School of Medicine, 251 E Huron Street, Feinberg 5-704, Chicago, Illinois 60611, USA, E-mail: ookocha@nm.org

Accepted: December 26, 2020

Published online: December 28, 2020

Citation: Okocha O, Luan-Erfe BM, Peace JM, et al. (2020) Intravenous Iron to Treat Anemia becomes an Essential Service to Conserve Blood during the COVID-19 Pandemic. J Clin Anesth Pain Manag 4(2):156-164 
Citation: Okocha O, Luan-Erfe BM, Peace JM, et al. (2020) Intravenous Iron to Treat Anemia becomes an Essential Service to Conserve Blood during the COVID-19 Pandemic. J Clin Anesth Pain Manag 4(2):156-164

transmission of the severe acute respiratory syndrome coronavirus 2, SARS-CoV-2, the donor pool will be further limited if SARS-CoV-2 is found to be transmissible through blood [5].

Blood banks are exploring ways to mitigate the need for blood components in the coming months. The Food and Drug Administration (FDA) issued an update loosening restrictions on individuals deemed at higher risk of transmitting human immunodeficiency virus, Creutzfeldt-Jakob Disease and malaria [2]. Nationwide, cancellations of elective surgeries is estimated to reduce the number of blood units transfused weekly by $25 \%$ [1]. This measure helps, but does not eliminate the ongoing demand for blood components. However, as the pandemic abates and previously deferred surgeries are once again being done, there may be a surge in demand. In addition, in an attempt to defer procedures and limit patient appointments, patients with ongoing or newly developed medical conditions with associated anemia may not be receiving treatments, such as endoscopy for ulcers, or hormonal or interventional therapies for dysfunctional uterine bleeding, that can eliminate blood loss. Therefore, they may present with more severe anemia. Patients with cancer, trauma, acute bleeds and hematologic disorders also continue to require blood transfusions.

In collaboration with the blood bank, our anemia clinic, embedded within the preoperative clinic and entirely managed by anesthesiologists, has expanded services to non-surgical patients and made concerted efforts to educate providers about our services. Efforts to identify patients with the potential need for blood components, the continued provision of efficient and effective diagnosis of treatable anemias and treatment with intravenous iron are considered essential services during this period of elective healthcare limitations [6]. Specifically, we are targeting surgical and obstetric patients, and other at-risk populations.

\section{Anemia}

Anemia is one of the most common conditions encountered in perioperative and non-perioperative settings. It is present in up to $75 \%$ of elective surgical patients [7], $30 \%$ of obstetric patients $[8,9]$, and $27.5 \%$ of emergency department patients [10]. Numerous studies have demonstrated an association between untreated anemia and increased morbidity and mortality. There is an increased risk of morbidity in patients even with mild-moderate anemia [11,12]. A hemoglobin of less than $8 \mathrm{~g} / \mathrm{dL}$ is associated with a 10-fold increase in mortality [11]. In obstetric patients, peripartum anemia is associated with numerous adverse neonatal outcomes including low birth weight, pre-term birth, neonatal anemia and postpartum depression [13].

Historically, blood transfusions are used as the default therapy for anemia in the perioperative period and for severe reductions in hemoglobin concentrations. However, several studies have shown that blood transfusions are associated with postoperative complications such as infections, need for intensive care and prolonged hospital length of stay $[14,15]$. In fact, the severity of anemia is directly correlated with worse outcomes, such as the likelihood of readmission within 30 days [16]. In obstetric patients, alloimmunization from exposure to antigens on transfused red blood cells is of particular concern. If a fetus carries these antigens, maternal antibodies can attack fetal red blood cells, causing hemolytic disease of the newborn, or even hydrops fetalis leading to intrauterine fetal demise [17]. Antepartum anemia is a leading risk factor for postpartum blood transfusion [18]. With $11.4 \%$ of maternal mortality attributed to postpartum hemorrhage [19], blood component shortage may lead to an increase in poor outcomes, as seen in resource-limited settings [20]. This underscores the importance of using alternative means to improve hematologic parameters in pregnant patients. Furthermore, blood transfusions are only a temporizing therapy that does not treat the underlying cause of anemia or replete iron stores to allow the bone marrow to produce red blood cells. The half-life of red blood cells is 57-59 days [21], so inherently, if the underlying cause of anemia is not addressed, patients re-develop anemia and remain transfusion-dependent. In addition to adverse outcomes, blood transfusion is expensive, poorly reimbursed and is a financial burden on hospitals [22,23].

The correlations among anemia, blood transfusions and associated negative outcomes have led to several studies on the treatment of anemia. Many have advocated for and developed patient blood management programs $[24,25]$. These efforts encourage healthcare providers to minimize blood transfusions by employing strategies to optimize hematopoiesis, reduce blood loss, and optimize anemia tolerance $[26,27]$.

Anemia is a serious but treatable medical condition, rather than simply an often-ignored laboratory abnormality. Anemia should be considered an indication for rescheduling surgery until fully evaluated and ideally treated [28]. Common underlying causes of readily treatable anemia are iron or vitamin $\mathrm{B}_{12}$ deficiencies. Associated conditions contributing to anemia include hypothyroidism, malabsorption, malnutrition, blood loss and chronic kidney disease. Worldwide, iron deficiency is the most common etiology of anemia [7] including in the obstetric population [29]. A recent study showed that $37 \%$ of surgical patients had anemia, $70 \%$ of which were diagnosed with iron deficiency [30]. Treatment is especially beneficial in the elderly, pregnant patients, those patients having surgeries associated with significant blood loss, patients who refuse blood transfusions, and when perioperative anticoagulation is planned. It is important to reiterate that anemia, like any other disease, should be treated. Addressing chronic conditions can improve both perioperative and long-term outcomes [30].

Several studies have evaluated the use of oral and intravenous iron or erythropoietin stimulating agents to treat anemia in the perioperative period. Intravenous iron is significantly more effective and better tolerated than oral iron supplements in treating iron deficiency anemia (IDA) [6]. Iron infusion is a safe and well-recognized treatment for IDA in pregnant patients, particularly during late pregnancy [31,32]. Currently available intravenous iron preparations are safer and better tolerated than older intravenous formulations [33-35]. In a German study, administration of intravenous 


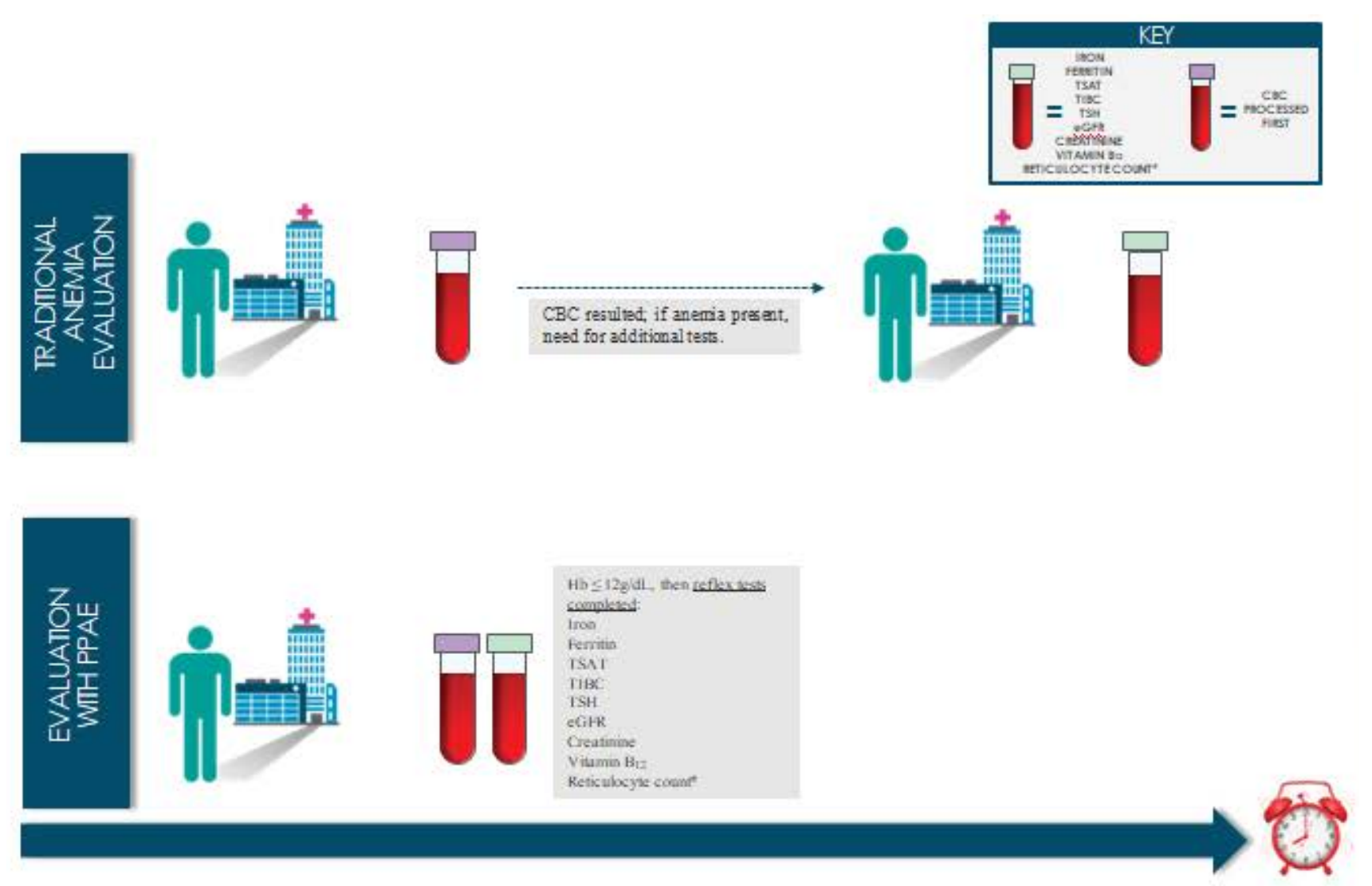

Figure 1: Diagram depicting time needed for anemia evaluation using pre-procedure anemia evaluation compared to current norms [30].

CBC: Complete blood count; eGFR: Estimated glomerular filtration rate; Hb: Hemoglobin; PPAE: Pre-procedure anemia evaluation; TIBC: Total iron binding capacity; TSAT: Transferrin saturation; TSH: Thyroid stimulating hormone; \#Reticulocyte count is obtained from the lavender-top blood vial.

iron showed decreased red blood cell transfusions in a select group of surgical patients. A limitation of that study is that patients were assessed a median of three days before surgery [36]. Although the optimal effect of iron infusion is seen at 3-4 weeks post-infusion [36], individual patient response to intravenous iron can vary based on bone marrow capacity. In one study of intravenous iron, $97 \%$ of patients doubled their reticulocyte counts within 48 hours of the infusion, with an increase in hemoglobin concentrations of 0.5 to $2.4 \mathrm{~g} / \mathrm{dL}$ by post-infusion day 7 [30,37]. Another study showed an average increase of $2.5 \mathrm{~g} / \mathrm{dL}$ among pregnant women 6 weeks after iron infusions [30]. However, one of the barriers to treatment of IDA is timely diagnosis.

Significant blood loss leads to more severe anemia. The use of tranexamic acid as an antifibrinolytic has been studied in the trauma and obstetric populations to limit hemorrhage. Although tranexamic acid and erythropoietin are associated with the risk of venous thromboembolism in some settings [38,39], this is far from universal as tranexamic acid has demonstrated safety in many patient populations $[40,41]$.

\section{Anemia Evaluation}

At our institution, one of the authors (BJS) developed a testing protocol that allows for diagnosis and evaluation of common etiologies of anemia in one visit. The anemia test- ing protocol, the pre-procedure anemia evaluation (PPAE), was implemented in January 2017, and has been shown to effectively and efficiently diagnose anemia including IDA [30], thereby allowing for expedited treatment (Figure 1). The PPAE order set includes reflex anemia testing when a hemoglobin concentration is less than or equal to $12 \mathrm{~g} / \mathrm{dL}$ on the complete blood count. Although the World Health Organization defines anemia as a hemoglobin less than $13 \mathrm{~g} / \mathrm{dL}$ for men and less than $12 \mathrm{~g} / \mathrm{dL}$ for women [42], we had designed the hemoglobin trigger for reflex testing on our PPAE at $12 \mathrm{~g} /$ $\mathrm{dL}$ or lower to simplify the process [30]. The diagnostic tests in the reflex testing evaluate the different etiologies of anemia and include reticulocyte count, serum iron concentration, transferrin saturation, serum ferritin concentration, total iron-binding capacity, thyroid-stimulating hormone concentration, creatinine concentration, estimated glomerular filtration rate and vitamin $B_{12}$ concentration. The PPAE process has been described in detail elsewhere [30]. Using the PPAE to diagnose anemia limits patient visits to a single appointment, eliminating the need for patients to return for further testing. It is even possible to diagnose anemia, delineate the underlying cause and provide treatment with just one patient visit. This is especially important during the COVID-19 pandemic.

\section{Treatment of Anemia}

IDA is diagnosed when an anemic patient has a serum fer- 
Citation: Okocha O, Luan-Erfe BM, Peace JM, et al. (2020) Intravenous Iron to Treat Anemia becomes an Essential Service to Conserve Blood during the COVID-19 Pandemic. J Clin Anesth Pain Manag 4(2):156-164

Table 1: Intravenous iron products, recommended dosing, adverse effects and costs [44,63-65,69].

\begin{tabular}{|c|c|c|c|}
\hline Iron preparation & Dose & Adverse effects & Drug cost per $1000 \mathrm{mg}^{\mathrm{a}}$ \\
\hline Iron sucrose & $\begin{array}{l}200 \text { to } 300 \text { mg every } 2 \text { days } \\
\text { Infusion time: } 15 \text { minutes }\end{array}$ & $\begin{array}{l}\text { Headache, muscle cramps, vomiting, diarrhea, } \\
\text { nausea }\end{array}$ & $\$ 300-400$ \\
\hline Ferric carboxymaltose & $\begin{array}{l}750 \mathrm{mg} \text { (weight } \geq 50 \mathrm{~kg} \text { ) or } 15 \mathrm{mg} / \\
\mathrm{kg} \text { (weight }<50 \mathrm{~kg} \text { ) every } 7 \text { days } \\
\text { Infusion time: } 15 \text { minutes }\end{array}$ & $\begin{array}{l}\text { Nausea, transient hypertension, flushing, } \\
\text { hypophosphatemia, dizziness. }\end{array}$ & $\$ 800-1000$ \\
\hline Ferric gluconate & $\begin{array}{l}125 \text { mg every } 2 \text { days } \\
\text { Infusion time: } 1 \text { hour }\end{array}$ & $\begin{array}{l}\text { Nausea, vomiting, diarrhea, hypotension, } \\
\text { muscle cramps, hypertension, dizziness, } \\
\text { dyspnea, chest pain }\end{array}$ & $\$ 150-250$ \\
\hline Ferumoxytol & $\begin{array}{l}510 \mathrm{mg}, 3-8 \text { days apart } \\
\text { Infusion time: } 15 \text { minutes }\end{array}$ & $\begin{array}{l}\text { Diarrhea, headache, nausea, dizziness, } \\
\text { hypotension, constipation, peripheral edema }\end{array}$ & $\$ 1800-2000$ \\
\hline $\begin{array}{l}\text { Low molecular weight } \\
\text { iron dextran }\end{array}$ & $\begin{array}{l}100 \mathrm{mg} \text { daily }(20 \mathrm{mg} / \mathrm{kg} \text {, maximum } \\
1000 \mathrm{mg} \text { ) } \\
\text { Infusion time: } 1 \text { hour }\end{array}$ & $\begin{array}{l}\text { Hypersensitivity reaction, nausea, vomiting, } \\
\text { injection site thrombosis or phlebitis }\end{array}$ & $\$ 300-400$ \\
\hline Ferric derisomaltose & $\begin{array}{l}1000 \mathrm{mg} \text { (weight } \geq 50 \mathrm{~kg} \text { ) or } 20 \\
\mathrm{mg} / \mathrm{kg} \text { (weight }<50 \mathrm{~kg} \text { ) Infusion } \\
\text { time: } 20 \text { minutes }\end{array}$ & Rash, nausea, hypophosphatemia & Not available ${ }^{c}$ \\
\hline
\end{tabular}

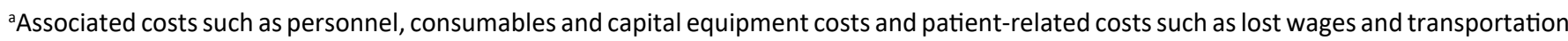
not included; 'Test dose recommended before first therapeutic dose; 'Approved in the United States February 2020, data not available.

\section{SAMPLE IRON INFUSION ORDER SET}

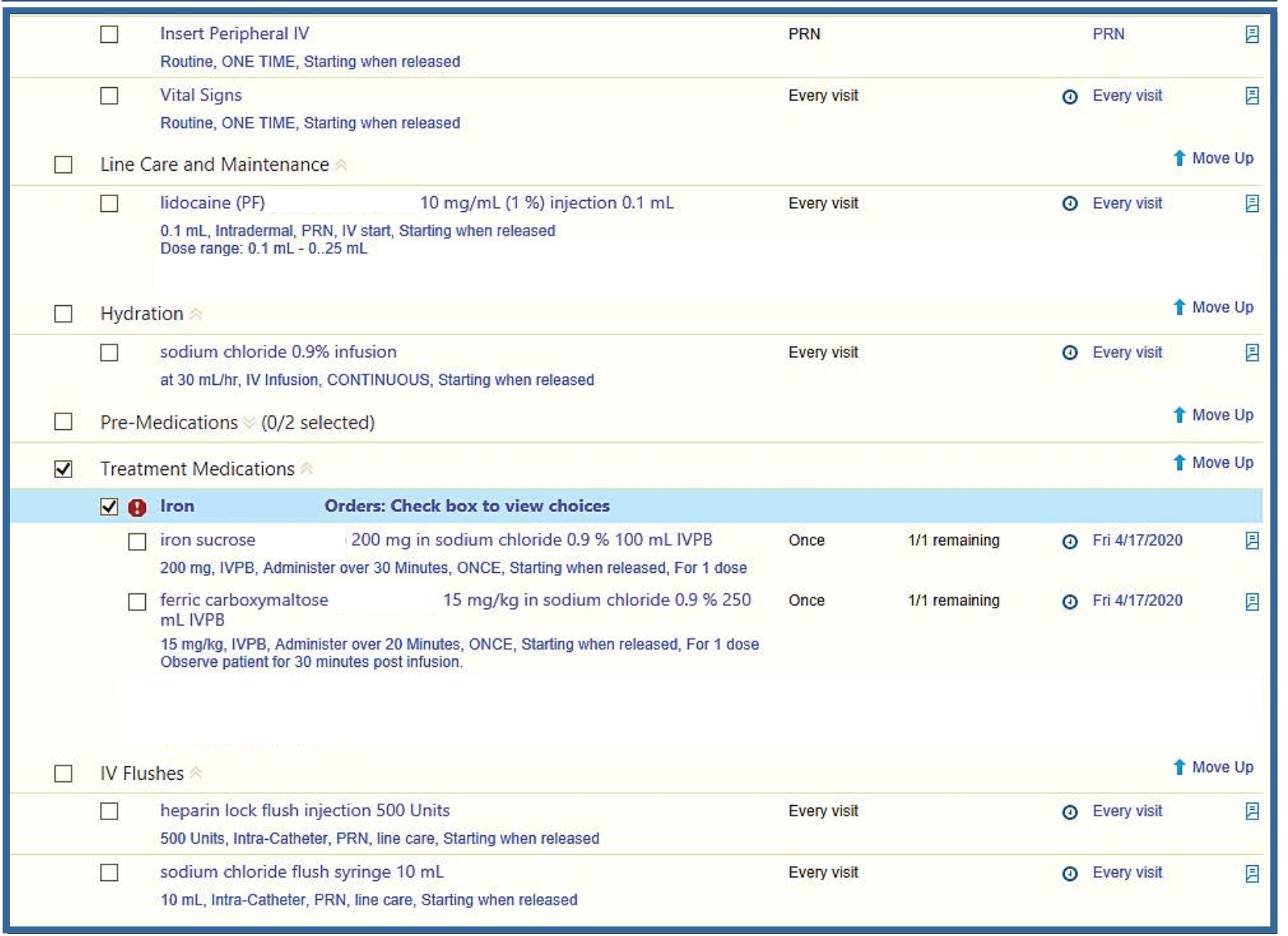

Figure 2: Sample order set for intravenous iron therapy. 
Citation: Okocha O, Luan-Erfe BM, Peace JM, et al. (2020) Intravenous Iron to Treat Anemia becomes an Essential Service to Conserve Blood during the COVID-19 Pandemic. J Clin Anesth Pain Manag 4(2):156-164

ritin concentration less than $30 \mathrm{ng} / \mathrm{mL}$ or transferrin saturation less than $20 \%$ [43]. When IDA is diagnosed, evaluation of underlying conditions causing IDA is necessary. Algorithms for determining etiology have been described elsewhere [44]. Our preoperative clinic processes provide guidance to address medical conditions that require follow up. We either initiate and complete appropriate diagnostics and therapies, or partner with primary care physicians and specialists. Timely identification of IDA and treatment with intravenous iron to optimize hemoglobin and prevent transfusions are recommended [45].

The current practice at our institution utilizes the PPAE to evaluate patients suspected of being anemic, pregnant patients or those planning procedures with significant blood loss. Within hours, iron deficiency can be determined, allowing for timely treatment with iron infusion [30]. Various iron preparations are available (Table 1 ). Figure 2 shows a sample order set for intravenous iron therapy. The dose of intravenous iron is based on the calculated iron deficit using the Ganzoni formula:

Total iron dose $(\mathrm{mg}$ iron) = lean body weight $(\mathrm{kg})$ (target hemoglobin - actual hemoglobin) (g/dL) $2.4+500 \mathrm{mg}$

Vitamin $B_{12}$ is administered if pernicious anemia is diagnosed and erythropoietin injections are given when chronic kidney disease or anemia of chronic disease is present, and in select circumstances such as for patients who refuse blood [46].

\section{Safety of Intravenous Iron}

Intravenous iron is a safer alternative than blood transfusions in iron-responsive anemia in light of the myriad of life-threatening acute complications from acute hemolytic transfusion reaction, transfusion-related acute lung injury and transfusion-associated anaphylaxis and infection $[47,48]$. Intravenous iron is also a more socially and economically responsible treatment of anemia given the current blood shortage and pandemic. There have been no demonstrable increase in rates of infection, severe gastrointestinal, cardiovascular, neurologic, respiratory or thromboembolic adverse events in patients given intravenous iron [49]. Ferric carboxymaltose is safe even at higher doses than available with other preparations, allowing for fewer clinic visits [50,51]. Ferric carboxymaltose is particularly favorable in this COVID-19 pandemic as total iron deficits can be replenished with only one or two visits. For example, a $750 \mathrm{mg}$ weekly dose of ferric carboxymaltose, in contrast to the $200 \mathrm{mg}$ alternate day dosing of iron sucrose, reduces the number of clinic visits from 8 to 2 , in a patient with an iron deficit of $1500 \mathrm{mg}$.

In the past, there was a relatively high risk of anaphylaxis with older, high molecular weight dextran iron infusions. The carbohydrate shell that stabilizes the iron core to prevent pain

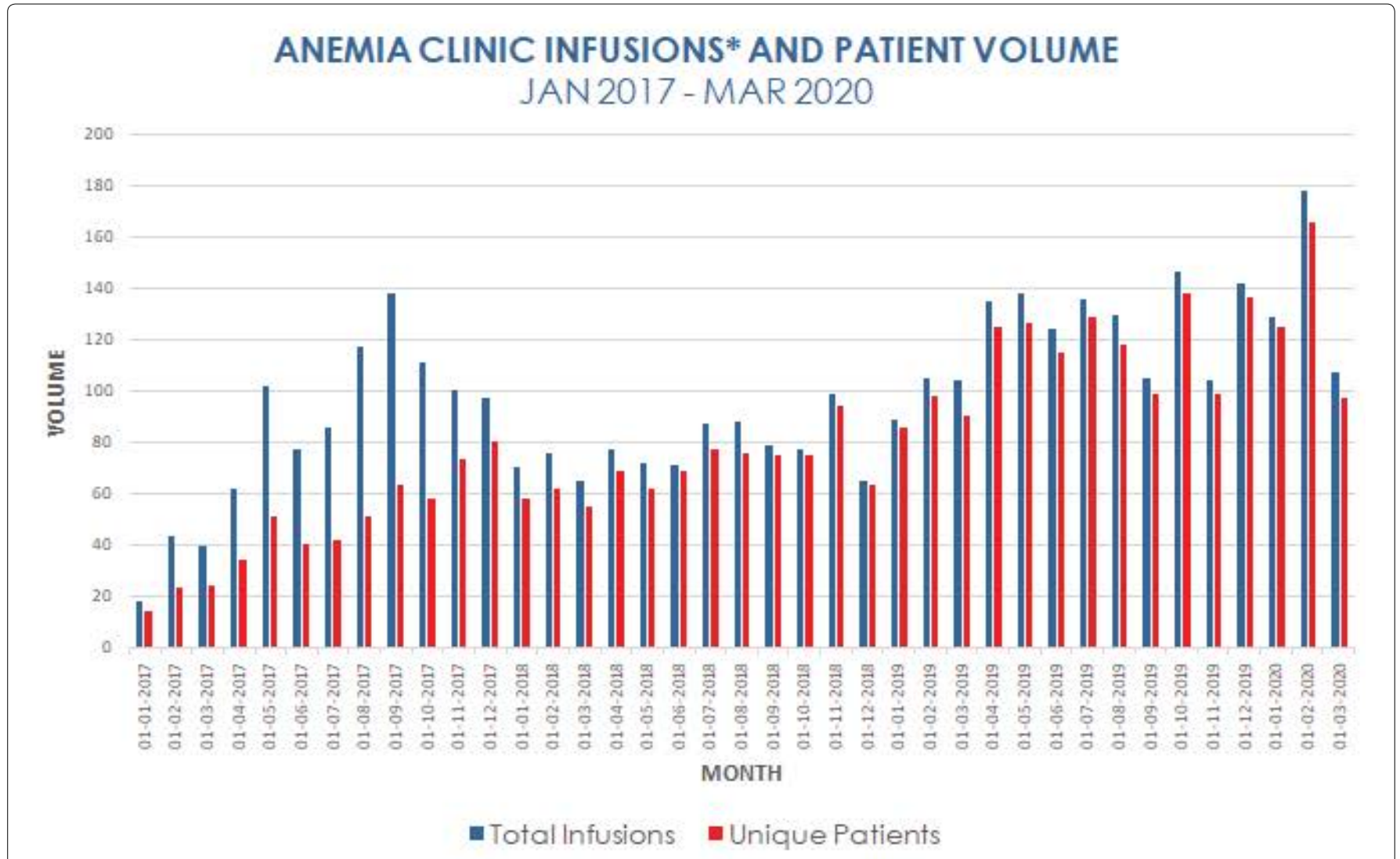


with infusion and hemodynamic instability from rapid release of elemental iron have been implicated in infusion reactions ranging from anaphylaxis to a mild infusion reaction characterized by rash, myalgias, dizziness and palpitations without ventilatory or hemodynamic compromise [52]. However, infusion reactions are rare with current preparations [49]. Among the older formulations, iron sucrose has the lowest rate of anaphylaxis and continues to be used widely today due to its safety profile and cost [53]. Ferric carboxymaltose, with the most stable carbohydrate shell among the newer formulations, has the lowest rate of anaphylaxis. Other complications of intravenous iron infusions include skin staining from extravasation and hypophosphatemia. Painless, but potentially permanent, light brown skin staining has been reported [5458]. Flushing the cannula with normal saline before and af- ter infusion, and checking for site infiltration helps avoid skin staining $[54,55]$. Laser treatments can treat the stains without subsequent hypopigmentation or scarring [56]. Intravenous iron can cause hypophosphatemia within 1-3 weeks $[49,59]$ as a result of renal phosphate wasting [60]. Two studies show that only ferric carboxymaltose causes severe hypophosphatemia, while iron sucrose causes mild hypophosphatemia of no clinical significance. The only consequence of hypophosphatemia in these studies was persistent fatigue [61-63]. Other, more severe consequences of hypophosphatemia such as cardiac arrhythmias may theoretically develop but have not been reported. High-risk patients should be monitored for low serum phosphate concentration and repletion with phosphate supplements as indicated [62]. In the last three years, our anemia clinic has safely administered more than 3,700

\section{SAMPLE ANEMIA CLINIC DISCHARGE SUMMARY}

If you have any questions or non-emergency concerns related to your iron infusion, please contact the Preoperative Clinic at XXX-XXX-XXXX between 7:30am and 5:30pm (Monday - Friday). If this is an emergency, visit your nearest emergency department or call 911.

Please understand that this content is intended to be used as supplemental information and does not substitute for the advice of your physician.

Today you received an iron infusion. Sometimes this medication can cause a decrease in your phosphate levels. This typically occurs 1-3 weeks after the infusion.

\section{Symptoms that may indicate your phosphorus level is too low include:}

- Changes in your mental state (for example, anxiety, irritability, or mild confusion)

- Bone issues, such as pain, fragility

- Fatigue

- Loss of appetite

- Muscle weakness

We recommend you increase phosphorus in your diet by consuming:

- Protein-rich foods such as

- Meats (especially white/light as opposed to dark chicken, turkey and pork)

- Fish

- Nuts

-Whole grains (wheat, oats, rice)

- Quinoa

- Sunflower and pumpkin seeds

- Peas, beans, lentils

- Soy

- Dairy products

- Chocolate

- Colas 
Citation: Okocha O, Luan-Erfe BM, Peace JM, et al. (2020) Intravenous Iron to Treat Anemia becomes an Essential Service to Conserve Blood during the COVID-19 Pandemic. J Clin Anesth Pain Manag 4(2):156-164

intravenous iron infusions (Figure 3), with only one patient reporting skin staining. When ferric carboxymaltose is administered, patients are warned about hypophosphatemia and phosphate-rich diets are recommended (Figure 4).

Of note, low molecular weight iron dextran is a formulation with a recommended dosing regimen that requires multiple visits to effectively replete iron stores since large intravenous doses have been associated with an increased incidence of adverse effects [63-65]. However, two studies suggest that doses of $1000 \mathrm{mg}$ can be given in a single visit, though this is not FDA approved, and they reported a not insignificant number of adverse events [64,65].

\section{Efficacy of Intravenous Iron}

Iron deficiency anemia can be treated with oral iron which is inexpensive and readily available; however, oral iron therapy requires several weeks to months to have an effect. Oral iron is rarely effective because compliance with treatment is low due to patients' inability to tolerate oral iron preparations [30]. Furthermore, even when patient adherence to oral iron therapy is confirmed, oral iron is less effective than intravenous iron in improving hemoglobin concentration [66]. Intravenous iron infusions are used to expeditiously raise hemoglobin concentrations before surgery, during pregnancy and before delivery. This strategy can be applied to all hospitalized and ambulatory patients with IDA as a blood conservation strategy during blood shortages. Numerous studies have shown robust hemoglobin responses as high as $1.55 \mathrm{~g} / \mathrm{dl}$ within two weeks even among patients with cancer in whom the etiology of anemia is multifactorial $[45,66,67]$. A randomized controlled trial of anemic patients undergoing elective cardiac surgery goes even further to show that a combination treatment using ferric carboxymaltose, erythropoietin, folic acid, and vitamin $B_{12}$ just one day before surgery reduced blood transfusions [68]. Thus, iron infusion can rapidly increase hemoglobin and decrease the need for blood transfusions for patients with IDA to preserve our blood bank supply. Specific examples at our institution where intravenous iron effectively raised hemoglobin concentrations within a short timeframe are numerous. A few examples of patients with significant elevations in hemoglobin concentrations within days after intravenous iron infusions are shown in Table 2. Data presented in Table 2 is from institutional review board (IRB)-approved studies that include subsets of anemic obstet- ric and surgical patients treated in our anemia clinic with intravenous iron.

\section{Conclusion}

The COVID-19 pandemic has significantly impacted our national blood supply. Prompt and effective treatment of anemia conserves resources. With the poor outcomes and significant financial burden associated with blood transfusion, and the ineffective treatment of IDA using oral iron, we argue that intravenous iron should be considered first-line therapy [22]. Although outpatient intravenous iron administration with newer iron preparations may be expensive, this practice is a cost-effective treatment of IDA since one must consider costs beyond simply the drug itself. The repeat visits required with the less-expensive, older iron formulations carry significant health system costs related to supplies, equipment and personnel and patient-related costs such as number of venipunctures and lost time from work and family [22].

Intravenous iron is the most efficient treatment of IDA. Streamlined testing utilizing a protocol such as the PPAE allows for prompt diagnosis of anemia, and determination of iron deficiency if present. Diagnosis and treatment can be completed with only 1-2 patient encounters and has long term benefits. This strategy reduces workload on healthcare providers, limits exposure of patients to SARS-CoV-2, and conforms to compliance with travel restrictions. Therefore, we advocate that iron infusions are essential, time-sensitive medical treatments that warrant implementation and continuation by providers in both surgical and non-surgical specialties during the COVID-19 pandemic. Although the FDA has issued updated guidance aimed at acutely increasing the potential donor pool, the safety of this practice is yet to be determined. The uncertainty of the severity and duration of the pandemic, and the reluctance of individuals to leave their homes suggest that reductions in our blood supply may persist for some time. We encourage institutions and providers to adopt tests such as the PPAE for timely diagnosis of IDA, especially in advance of resumption of elective surgeries or ongoing procedures with high blood utilization, and to institutionalize the use of intravenous iron therapy for IDA. We recommend implementing these changes not only for the current COVID-19 blood shortage, but because future resource limitations are unpredictable.

Table 2: Characteristics of sample patients with significant elevations in hemoglobin concentrations following intravenous iron infusions ${ }^{\mathrm{a}}$.

\begin{tabular}{|c|c|c|c|c|c|c|c|c|}
\hline $\begin{array}{l}\text { Age } \\
\text { (yrs) }\end{array}$ & Sex & ASA-PS & Comorbidities & Procedure & $\begin{array}{l}\text { Initial Hb } \\
\text { (g/dL) }\end{array}$ & $\begin{array}{l}\text { Post iron } \\
\text { infusion } \mathrm{Hb} \\
(\mathrm{g} / \mathrm{dL})\end{array}$ & $\begin{array}{l}\text { Hb change } \\
(\mathrm{g} / \mathrm{dL})\end{array}$ & $\begin{array}{l}\text { Time interval } \\
\text { (days) }\end{array}$ \\
\hline 33 & $\mathrm{~F}$ & 2 & Fibroids & Myomectomy & 7.4 & 9.5 & 2.1 & 14 \\
\hline 45 & $\mathrm{~F}$ & 3 & $\begin{array}{l}\text { Lung cancer, } \\
\text { Myasthenia gravis }\end{array}$ & Lung lobectomy & 10.2 & 12.6 & 2.4 & 15 \\
\hline 40 & $\mathrm{~F}$ & 2 & Pregnancy & Vaginal delivery & 8.5 & 10.8 & 2.3 & 19 \\
\hline 37 & $\mathrm{~F}$ & 2 & Twin pregnancy & Vaginal twin delivery & 9.6 & 11.4 & 1.8 & 10 \\
\hline 31 & $\mathrm{~F}$ & 2 & Gastric bypass & Cesarean delivery & 8.1 & 9.7 & 1.6 & 14 \\
\hline
\end{tabular}

ASA-PS: American Society of Anesthesiologists Physical Status; F: Female; Hb: Hemoglobin.

${ }^{a}$ No reported adverse effects or need for blood transfusions. 
Citation: Okocha O, Luan-Erfe BM, Peace JM, et al. (2020) Intravenous Iron to Treat Anemia becomes an Essential Service to Conserve Blood during the COVID-19 Pandemic. J Clin Anesth Pain Manag 4(2):156-164

\section{Acknowledgments}

The authors acknowledge Jeffrey Jameel, M.D., Northwestern Memorial Hospital, Chicago, Illinois, for his contribution to Figures 1 and Figure 3.

Dr. Okocha: This author helped with conceptualization, writing the original draft, reviewing and editing.

Dr. Luan-Erfe: This author helped with writing the original draft, reviewing and editing.

Dr. Peace: This author contributed writing to the original draft, reviewing and editing.

Dr. Sweitzer: This author helped with conceptualization, reviewing and editing.

\section{Funding}

None.

\section{Conflicts of Interest}

None.

\section{References}

1. American Red Cross (2020) Keeping our hospital partners informed during novel coronavirus (COVID-19) pandemic.

2. U.S. Food and Drug Administration (2020) Coronavirus (COVID-19) update: FDA provides updated guidance to address the urgent need for blood during the pandemic.

3. Gurevitz SA (2011) Update and utilization of component therapy in blood transfusions. Lab Med 42: 235-240.

4. American Red Cross (2020) Frequently asked questions.

5. Chang L, Yan Y, Wang L (2020) Coronavirus disease 2019: Coronaviruses and blood safety. Transfus Med Rev 34: 75-80.

6. Shander A, Goobie SM, Warner MA, et al. (2020) Essential role of patient blood management in a pandemic: A call for action. Anesth Analg 131: 74-85.

7. Munting KE, Klein AA (2019) Optimisation of pre-operative anaemia in patients before elective major surgery - why, who, when and how? Anaesthesia 74: 49-57.

8. McLean E, Cogswell M, Egli I, et al. (2009) Worldwide prevalence of anaemia, WHO vitamin and mineral nutrition information system, 1993-2005. Public Health Nutr 12: 444-454.

9. Mei Z, Cogswell ME, Looker AC, et al. (2011) Assessment of iron status in US pregnant women from the national health and nutrition examination survey (NHANES), 1999-2006. Am J Clin Nutr 93: 1312-1320.

10. Beverina I, Brando B (2019) Prevalence of anemia and therapeutic behavior in the emergency department at a tertiary care hospital: Are patient blood management principles applied? Transfus Apher Sci 58: 688-692.

11. Baron DM, Hochrieser H, Posch M, et al. (2014) Preoperative anaemia is associated with poor clinical outcome in non-cardiac surgery patients. Br J Anaesth 113: 416-423.

12. Musallam KM, Tamim HM, Richards T, et al. (2011) Preoperative anaemia and postoperative outcomes in non-cardiac surgery: A retrospective cohort study. Lancet 378: 1396-1407.

13. Malinowski AK, D'Souza R, Khan KS, et al. (2019) Reported out- comes in perinatal iron deficiency anemia trials: A systematic review. Gynecol Obstet Invest 84: 417-434.

14. Ferraris VA, Davenport DL, Saha SP, et al. (2012) Surgical outcomes and transfusion of minimal amounts of blood in the operating room. Arch Surg 147: 49-55.

15. Li Y, Stocchi L, Rui Y, et al. (2015) Perioperative blood transfusion and postoperative outcome in patients with crohn's disease undergoing primary ileocolonic resection in the "biological era". J Gastrointest Surg 19: 1842-1851.

16. Koch CG, Li L, Sun Z, et al. (2017) From bad to worse: Anemia on admission and hospital-acquired anemia. J Patient Saf 13: 211216.

17. Webb J, Delaney M (2018) Red blood cell alloimmunization in the pregnant patient. Transfus Med Rev 32: 213-219.

18. Rouse DJ, MacPherson C, Landon M, et al. (2006) Blood transfusion and cesarean delivery. Obstet Gynecol 108: 891-897.

19. Creanga AA, Syverson C, Seed K, et al. (2017) Pregnancy-related mortality in the United States, 2011-2013. Obstet Gynecol 130: 366-373.

20. Bates I, Chapotera GK, McKew S, et al. (2008) Maternal mortality in sub-Saharan Africa: The contribution of ineffective blood transfusion services. BJOG 115: 1331-1339.

21. Shrestha RP, Horowitz J, Hollot CV, et al. (2016) Models for the red blood cell lifespan. J Pharmacokinet Pharmacodyn 43: 259274.

22. Quintana-Diaz M, Fabra-Cadenas $S$, Gomez-Ramirez $S$, et al. (2016) A fast-track anaemia clinic in the emergency department: Feasibility and efficacy of intravenous iron administration for treating sub-acute iron deficiency anaemia. Blood Transfus 14: 126-133.

23. Shander A, Hofmann A, Ozawa S, et al. (2010) Activity-based costs of blood transfusions in surgical patients at four hospitals. Transfusion 50: 753-765

24. Goel R, Cushing MM, Tobian AA (2016) Pediatric patient blood management programs: Not just transfusing little adults. Transfus Med Rev 30: 235-241.

25. Shander A, Javidroozi M, Lobel G (2017) Patient blood management in the intensive care unit. Transfus Med Rev 31: 264-271.

26. Goobie SM, Gallagher T, Gross I, et al. (2019) Society for the advancement of blood management administrative and clinical standards for patient blood management programs. 4th edition (pediatric version). Pediatr Anesth 29: 231-236.

27. WHO (2010) Availability, safety and quality of blood products. World Health Organization, Geneva.

28. Shander A, Van Aken H, Colomina MJ, et al. (2012) Patient blood management in Europe. Br J Anaesth 109: 55-68.

29. Esen UI (2017) Iron deficiency anaemia in pregnancy: The role of parenteral iron. J Obstet Gynaecol 37: 15-18.

30. Okocha O, Dand H, Avram MJ, et al. (2020) An effective and efficient testing protocol for diagnosing iron-deficiency anemia preoperatively [Published online ahead of print March 17 2020]. Anesthesiology 133: 109-118.

31. Govindappagari S, Burwick RM (2019) Treatment of iron deficiency anemia in pregnancy with intravenous versus oral iron: Systematic review and meta-analysis. Am J Perinatol 36: 366337. 
Citation: Okocha O, Luan-Erfe BM, Peace JM, et al. (2020) Intravenous Iron to Treat Anemia becomes an Essential Service to Conserve Blood during the COVID-19 Pandemic. J Clin Anesth Pain Manag 4(2):156-164

32. Bhatt R, Rao P, Sharma S, et al. (2019) Ferricarboxy maltose to treat iron deficiency anemia in pregnancy: Is it a feasible option? Int J Reprod Contracept Obstet Gynecol 8: 19-23.

33. Peters F, Ellermann I, Steinbicker AU (2018) Intravenous iron for treatment of anemia in the 3 perisurgical phases: A review and analysis of the current literature. Anesth Analg 126: 1268-1282.

34. Richards T, Clevenger B, Keidan J, et al. (2015) PREVENTT: Preoperative intravenous iron to treat anaemia in major surgery: Study protocol for a randomised controlled trial. Trials 16: 254.

35. Bisbe E, García-Erce JA, Díez-Lobo Al, et al. (2011) A multicentre comparative study on the efficacy of intravenous ferric carboxymaltose and iron sucrose for correcting preoperative anaemia in patients undergoing major elective surgery. $\mathrm{Br} J$ Anaesth 107: 477-478.

36. Ellermann I, Bueckmann A, Eveslage M, et al. (2018) Treating anemia in the preanesthesia assessment clinic: Results of a retrospective evaluation. Anesth Analg 127: 1202-1210.

37. Johnson CS (1979) Intravenous iron-dextran in the treatment of iron deficient anemia. J Natl Med Assoc 71: 1101-1105.

38. Myers SP, Kutcher ME, Rosengart MR, et al. (2019) Tranexamic acid administration is associated with an increased risk of posttraumatic venous thromboembolism. J Trauma Acute Care Surg 86: 20-27.

39. Singbartl G (1994) Adverse events of erythropoietin in long-term and in acute/short-term treatment. Clin Investig 72: S36-S43.

40. Shakur H, Roberts I, Fawole B, et al. (2017) Effect of early tranexamic acid administration on mortality, hysterectomy, and other morbidities in women with post-partum haemorrhage (WOMAN): An international, randomised, double-blind, placebo-controlled trial. Lancet 389: 2105-2116.

41. Shakur H, Roberts I, Bautista R, et al. (2010) Effects of tranexamic acid on death, vascular occlusive events, and blood transfusion in trauma patients with significant haemorrhage (CRASH-2): A randomised, placebo-controlled trial. Lancet 376: 23-32.

42. World Health Organization (2011) Haemoglobin concentrations for the diagnosis of anaemia and assessment of severity.

43. Goodnough LT, Shander A (2012) Patient blood management. Anesthesiology 116: 1367-1376.

44. Warner M, Shore-Lesserson L, Shander A, et al. (2020) Perioperative anemia: Prevention, diagnosis, and management throughout the spectrum of perioperative care. Anesth Analg 130: 1364-1380.

45. Theusinger OM, Leyvraz PF, Schanz U, et al. (2007) Treatment of iron deficiency anemia in orthopedic surgery with intravenous iron: Efficacy and limits: A prospective study. Anesthesiology 107: 923-927.

46. U.S. Food and Drug Administration (2017) Information on erythropoiesis-stimulating agents (ESA) epoetin alfa (marketed as Procrit, Epogen), darbepoetin alfa (marketed as Aranesp).

47. Despotis GJ, Zhang L, Lublin DM (2007) Transfusion risks and transfusion-related pro-inflammatory responses. Hematol Oncol Clin North Am 21: 147-161.

48. Rohde JM, Dimcheff DE, Blumberg N, et al. (2014) Healthcare-associated infection after red blood cell transfusion: A systematic review and meta-analysis. JAMA 311: 1317-1326.

49. Avni T, Bieber A, Grossman A, et al. (2015) The safety of intravenous iron preparations: Systematic review and meta-analysis. Mayo Clin Proc 90: 12-23.

50. Ehlken B, Nathell L, Gohlke A, et al. (2019) Evaluation of the reported rates of severe hypersensitivity reactions associated with ferric carboxymaltose and iron (iii) isomaltoside 1000 in Europe based on data from EudraVigilance and VigiBase ${ }^{\mathrm{TM}}$ between
2014 and 2017. Drug Saf 42: 463-471.

51. Neiser S, Rentsch D, Dippon U, et al. (2015) Physico-chemical properties of the new generation IV iron preparations ferumoxytol, iron isomaltoside 1000 and ferric carboxymaltose. Biometals 28: 615-635.

52. Girelli D, Ugolini S, Busti F, et al. (2018) Modern iron replacement therapy: Clinical and pathophysiological insights. Int J Hematol 107: 16-30.

53. Wang C, Graham DJ, Kane RC, et al. (2015) Comparative risk of anaphylactic reactions associated with intravenous iron products. JAMA 314: 2062-2068.

54. Crowley CM, McMahon G, Desmond J, et al. (2019) Skin staining following intravenous iron infusion. BMJ Case Rep 12: e229113.

55. Thompson J, Pavord S, Lim K (2014) Severe haemosiderin pigmentation after intravenous iron infusion. Intern Med J 44: 706708.

56. Pérez-Pevida B, Kamocka A (2018) Haemosiderin pigmentation after intravenous iron infusion. BMJ 360: 69.

57. Crowley CM, McMahon G, Desmond J, et al. (2020) Preventing skin staining: An effective iron infusion protocol. Int J Health Care Qual Assur.

58. Raulin C, Werner S, Greve B (2001) Circumscripted pigmentations after iron injections-treatment with Q-switched laser systems. Lasers Surg Med 28: 456-460.

59. Nataatmadja MS, Francis R (2020) Recurrent severe hypophosphatemia following intravenous iron administration. Clin Case Rep 8: 243-246.

60. Wolf M, Koch TA, Bregman DB (2013) Effects of iron deficiency anemia and its treatment on fibroblast growth factor 23 and phosphate homeostasis in women. J Bone Miner Res 28: 17931803.

61. Hardy S, Vandemergel $X$ (2015) Intravenous iron administration and hypophosphatemia in clinical practice. Int $\mathrm{J}$ Rheumatol 2015: 468675.

62. Tulewicz-Marti E, Moniuszko A, Korcz T, et al. (2018) P769 Hypophosphatemia in patients with inflammatory bowel disease after intravenous iron infusion: One centre prospective study. Journal of Crohn's and Colitis 12: S500.

63. (2019) INFeD ${ }^{\circledR}$ (Iron dextran injection usp). Allergan, Inc, Naperville, IL.

64. Auerbach M, Pappadakis JA, Bahrain H, et al. (2011) Safety and efficacy of rapidly administered (one hour) one gram of low molecular weight iron dextran (INFeD) for the treatment of iron deficient anemia. Am J Hematol 86: 860-862.

65. Wong L, Smith S, Gilstrop M, et al. (2016) Safety and efficacy of rapid $(1,000 \mathrm{mg}$ in $1 \mathrm{hr}$ ) intravenous iron dextran for treatment of maternal iron deficient anemia of pregnancy. Am J Hematol 91: 590-593.

66. Keeler BD, Simpson JA, Ng O, et al. (2017) Randomized clinical trial of preoperative oral versus intravenous iron in anaemic patients with colorectal cancer. Br J Surg 104: 214-221.

67. Wilson MJ, Dekker JW, Bruns E, et al. (2018) Short-term effect of preoperative intravenous iron therapy in colorectal cancer patients with anemia: Results of a cohort study. Transfusion 58: 795-803.

68. Spahn DR, Schoenrath F, Spahn GH, et al. (2019) Effect of ultrashort-term treatment of patients with iron deficiency or anaemia undergoing cardiac surgery: A prospective randomised trial. Lancet 393: 2201-2212.

69. U.S. National Library of Medicine (2020) DailyMed. 